\title{
EXPLORING THE EFFECT OF DEMOGRAPHIC ELEMENTS ON THE EVALUATION OF THE SCENIC BEAUTY OF VARIOUS LANDFORMS - PRELIMINARY RESULTS
}

\author{
A. TSOUCHLARAKI \\ Hellenic Open University, Greece.
}

\begin{abstract}
This article reports on a piece of research which attempts to take 32 different images of landforms and to show how different classes of people perceive or 'value' the scenic beauty of these landforms. This is an interesting question and one which bears research. In this case, the term 'scenic beauty' refers to the public preference of various forms of the earth's relief. It is a figure which, even though depends on various subjective factors, aims to quantify the general preference of the public for various landforms. A questionnaire survey takes place to investigate the public preference, using a sample of 221 persons in the area of Athens and Piraeus, and the city suburbs. This area concentrates a very large proportion of Greece's population and can ensure variety in terms of social and demographic status of the sampled population. The means used to demonstrate the various forms of reliefs are 32 digital relief images, created using an algorithm developed for this purpose. The representative selection of the sample of digital images took place after the classification of the forms of relief in Lefka Ori mountain range in Crete. The questionnaire comprised of 11 questions describing the person questioned in relation to the environment he/she has experienced or knows and his/her contact with the countryside. Each question corresponded to a factor that had been generalised in categories. Following this, a primary statistical analysis of variance was carried out for each of the factors examined and some preliminary results are reported. Little research in this specific area has been done and it is interesting to explore further the way people with different social backgrounds react or perceive the various landforms.

Keywords: analysis of variance, demographic elements, landforms, questionnaire, scenic beauty.
\end{abstract}

\section{INTRODUCTION}

Landscape is the combined result of physiography, geological formations, vegetation, waters and the various cultural interventions that occur in a given area. This combination attributes shape, line, colour and texture to a landscape, while the aesthetic result is considered on the basis of the variety or the uniqueness offered and is usually classified into three main classes: (1) indistinctive, (2) common, (3) distinctive [1,2]. This classification determines those landscapes which are most important and those which are of lesser value from the standpoint of scenic beauty. The classification is based on the premise that all landscapes have some scenic value, but those with the most variety or diversity have the greatest potential for high scenic beauty. A frame of reference is developed by which to judge the physical features of an area as indistinctive, common or distinctive. Features, such as landforms, water forms, rock formations and vegetative patterns are compared singularly or in combination. Through this comparison, an area's overall degree of scenic beauty and resultant variety class rating is determined. The various approaches developed for determining landscape visual quality refer to the general output of the synthesis of all physical variables of the environment, physiography, soil, vegetation, hydrological elements and not individually to each element. In these approaches, the visual quality of a landscape is evaluated either indirectly by thematic maps according to standardised criteria based on the experience of the scientists, or directly on the basis of psychometrical methods that quantify directly the public's preference by demonstrating through a certain means the landscape under evaluation [1, 3-9]. However, relief constitutes a part of these approaches and not the main objective.

(C) 2007 WIT Press, www.witpress.com

ISSN: 1744-3687 (paper format), ISSN: 1744-3679 (online), http://journals.witpress.com DOI: 10.2495/D\&N-V2-N3-247-257 
The physical relief is a variable of utmost significance in the course of the analysis, evaluation, design and management of the visual environment, owing to four main reasons:

1. It constitutes the three-dimensional background, the backbone on which all other physical variables develop (vegetation, water resources, etc.).

2. It is very difficult, if not impossible, and very costly to vary in shape, form and size.

3. The range of visibility from each observation point depends on the form of the earth's relief.

4. The spatial arrangement of many activities depends on the form, the shape and the individual characteristics of the relief.

In this sense, there are cases where it would be desirable to isolate the relief from the other elements which make up the visual environment and to investigate its scenic beauty separately. Such cases include the technical works that cause major and permanent alterations to the relief, as well as the works, of which the spatial arrangement depends on the morphology of the ground. Slopes, distances, hypsometric difference values, viewsheds are elements of the relief that affect the visual quality of a landscape, but also the ability of a particular landscape to accept and absorb new activities.

The experience of the scientists show that the more mountainous the form of the relief, the more distinct the landscape category offered $[1,6]$. It is interesting in this case to explore the way various persons react, based on different demographic elements, to the different forms of the relief. The way each observer evaluates any given landscape is a very complicated issue, a matter difficult to predict. Many factors can possibly influence this evaluation, ranging from factors that can be registered, such as the usual demographic elements, to imponderable factors, such as the mood of the observers at the time of the evaluation. Besides, what 'one likes or dislikes' does not remain constant with time. As a person matures, his/her attitude towards many things in life changes. Therefore it would be utopia to try to predict with precision the preference of an observer for a given landscape, but this is not the aim of this research. This paper presents some preliminary results of a research work that aims to investigate the way in which the various demographic elements of the persons questioned influence the preference in the case of the evaluation of the scenic beauty of a landform. Whatever conclusion can be drawn, even based on the few factors that can be registered, would be useful in the interpretation of the phenomenon.

\section{INVESTIGATION METHODOLOGY}

\subsection{Digital relief visualisation}

The investigation of the public preference to the various landforms can be achieved by using questionnaires, utilising some means for presenting the different forms of relief. In this case, it was not possible to use actual photographs as a means to demonstrate the different forms, because they would simultaneously provide information on the vegetation, the soil and the land uses of a given area, something that could influence the preference of the persons questioned. Therefore, the survey was put into effect using digital visual representations as a means to demonstrate the various forms of relief. These are images created using a Digital Terrain Model and the respective shading image. The creation of these images relied on an algorithm that had been developed for this purpose, producing the perspective image of the relief, as this would look like if it had been photographed from a known shooting point in relation to a given target, using a photo camera of known geometry [10]. All shootings are strictly horizontal, considering the geometry of a $35 \mathrm{~mm}$ camera with a normal lens $(f=50 \mathrm{~mm})$ and a predetermined data analysis scheme. 
The study area was the Lefka Ori mountain range in Crete, owing to variety of forms of relief it includes. The IDRISI GIS package was used for processing of information, as well as creation of a DTM and of other derivative elements.

The representative selection of the sample of the digital representations that would be included in the questionnaire took place following a classification of the relief forms, based on a method developed for this purpose, which is a modification of Hammond's classification method. Hammond's classification was selected as the basis, first, because it is one of the most acknowledged relief classification methods, and, second, because it makes use of a few variables in the quantitative determination of forms, a feature that is necessary to have a limited and logical number of categories. According to Hammond's classification, to classify large areas, the study area is sub-divided into large rectangular parts, while the three quantitative parameters used are based on their definition on the main stages of geomorphic process [11]. These parameters are:

1. The percentage of flat slopes. Slopes up to $8 \%$ are defined as flat.

2. The maximum hypsometric difference observed within the rectangular part.

3. The percentage of flat slopes that is observed at the upper or lower half of the hypsometric difference range.

The basic characteristic of the classification method that was developed for the purpose of this study is that it aims to predict perspective observation conditions [12]. In this classification method, the three parameters of Hammond's method are adopted, while one more parameter is added with regard to the observation post. The observation post is distinguished as superior, equal or inferior, depending on the relative elevation position of the observer and the objects he observes and plays an important role in the general perception of the shapes and forms [13]. The four parameters are not applied to static rectangular parts, as in Hammond's classification, but to moving windows of particular dimensions and a specified movement increment on the digital terrain model, while the four main orientations are examined, i.e., north, south, east and west towards the centre of the moving windows.

The classification resulted in 32 relief categories. A horizontal position was selected for each category, for creating a digital representation. The 32 images created are representative of the different forms of relief in the study area and are depicted in Fig. 1. As far as the scenic beauty is concerned, the persons questioned were asked to rate it on a 1-10 scale (1 representing a very small preference concerning the scenic beauty of the landform and 10 representing a high preference). This scale was chosen, because the studies of landscape aesthetics which use photographic imagery (both actual and simulated) and 1-10 scales for response are common in use.

\subsection{Questionnaire design and execution}

There are many rules for the design and execution of a questionnaire and also many decisions that have to be taken $[14,15]$. The size of the sample, the sampling methodology, the form and type of questionnaire, the duration of a questionnaire are some of the matters that have to be examined. This subsection is devoted to these matters.

The questionnaire was used in the area of Athens, Piraeus and the city suburbs, using a sample of 221 persons. This area, comprising of 52 municipalities and 5 communities, offered the following advantages:

(A) It is an area of Greece's capital that concentrates a very large proportion of the population and is suitable for ensuring variety in terms of the different social and demographic characteristics of the persons questioned. 


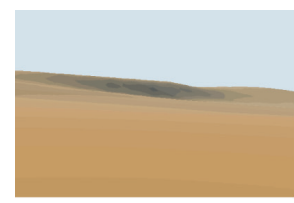

1

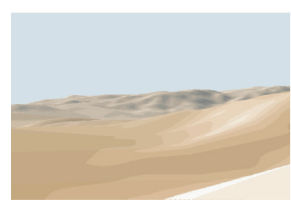

5

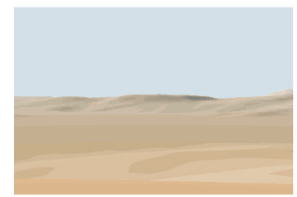

9

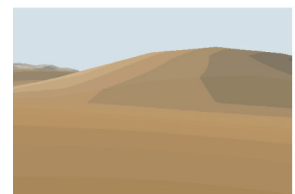

13

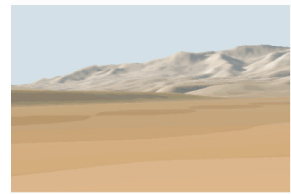

17

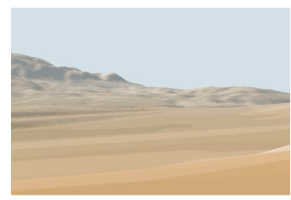

21

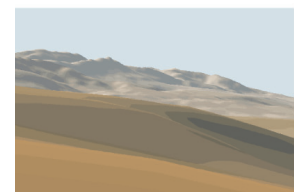

25

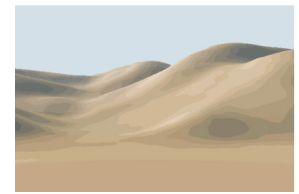

29

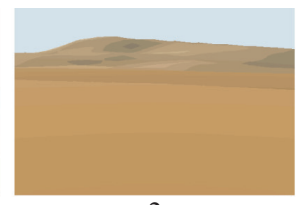

2

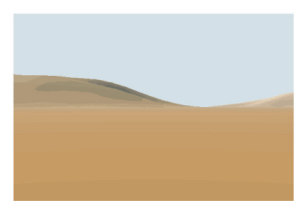

6

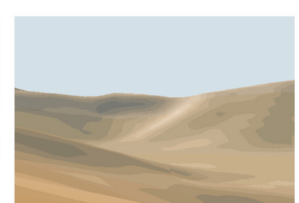

10

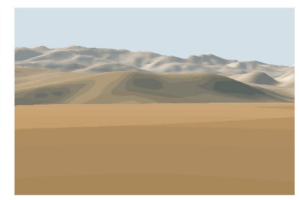

14

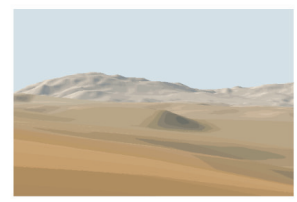

18

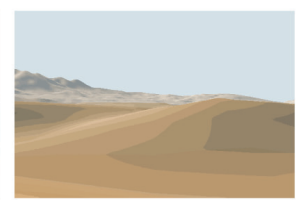

22

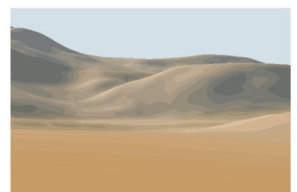

26

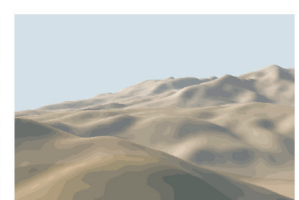

30

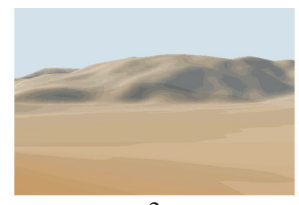

3

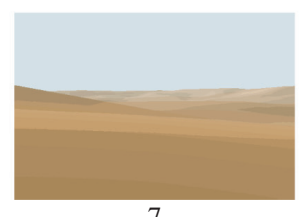

7

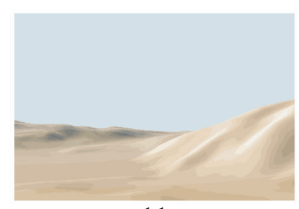

11

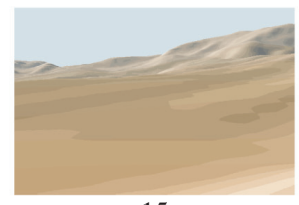

15

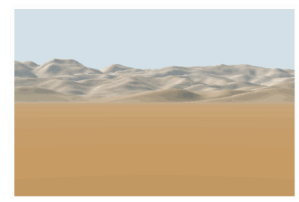

19

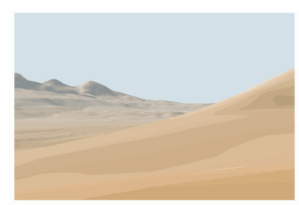

23

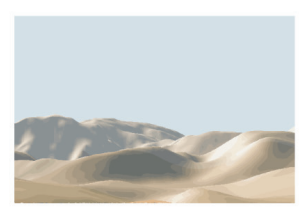

27

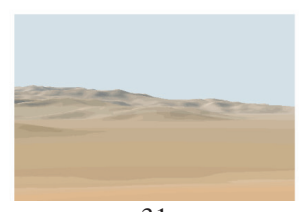

31

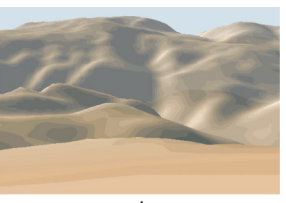

4

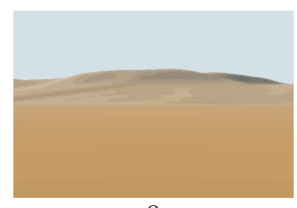

8

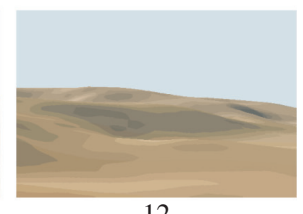

12

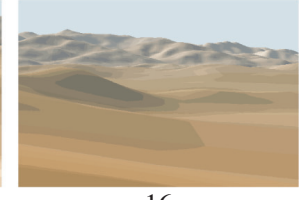

16

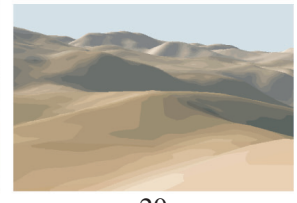

20

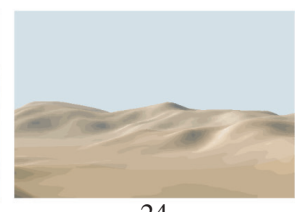

24

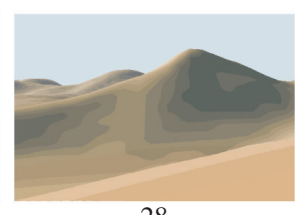

28

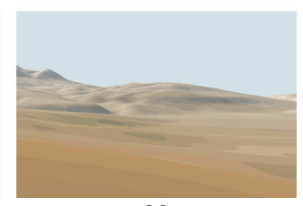

32

Figure 1: The 32 images of landforms included in the questionnaire. 
(B) The population of Greece's capital comes mostly from different areas of Greece, and allows the inclusion of people from the provinces, with different experiences and mental representations of the various forms of the relief.

The total population of the study area is 3,020,562. The area includes both rich and poor quarters, both densely and scarcely populated, all of them being within the city plan limits so that they can be mapped and can facilitate the organisation and implementation of the questionnaire.

A stratification of the municipalities of Athens was effected before the selection of the persons to be questioned. To ensure the representation of the entire population, two individual layers were used before adopting the final sampling. For the first layering, the municipalities were classified into three categories, according to their population: small $(X<30,000$ inhabitants), average $(30,000<X<$ $70,000)$ and big $(X>70,000$ inhabitants). For the second layering, we used the existing classification of the prefectures: Athens, Eastern Attica, Western Attica and Piraeus. Thus, we thus created $3 \times 4=$ 12 layers.

In a simple random sampling, the sample size was calculated as follows [14]:

$$
P[|\bar{y}-\bar{Y}|<d]>1-a,
$$

where $\bar{Y}$ is the population average of the requisite characteristic, which in this case is the visual value; $\bar{y}$ is the population average estimate derived from the sample; $d$ is the error margin or the desired measurement accuracy of the average; $1-a$ is the confidence coefficient.

It is clear from eqn (1) that the size of the sample $n$ is determined by the following formula [14]:

$$
n=\frac{n_{0}}{1+\frac{n_{0}}{N}},
$$

in which:

$$
n_{0}=\left(\frac{s z_{a / 2}}{d}\right)^{2}
$$

where $N$ is the total population; $s^{2}$ is the dispersion of the characteristic; $z_{a / 2}$ is the upper $a / 2$-point of the normal distribution $N(0,1)$.

The term $n_{0} / N$, when the value of $N$ is very big, tends towards zero and is ignored. In the layered sampling analysis, the same reasoning is followed, with the exception being that eqns (2) and (3) are applied in each layer, using the respective dispersion of the characteristic which each layer exhibits. In case the dispersion is unknown, we can use its value from previous studies related to the subject matter [14].

Because of the fact that there are no previous studies related to certain subject matter, we used the results of an experimental implementation on 55 students of the second and eighth semesters of the Department of Rural and Surveying Engineering of the National Technical University of Athens. This implementation was a simple questionnaire including the 32 images and asking students to evaluate them on a 1-10 scale, according to their preference. In this pilot study, the previous experience of the scientists came true as the more mountainous the form of the relief, the higher the values that resulted from the students' preference. The results of this implementation showed that the standard deviation differs among images and ranges from 1.64 to 2.75 units. To determine the final sample, 
we assumed that all layers present the same dispersion and took the worst case to be the standard deviation: $s=2.75$ units. The desired measurement accuracy of the mean was determined to be $d=0.40$, a value lower than the unit half. Therefore, for a $95 \%$ probability, the application of the above formulae shows that the size of the sample is:

$$
n=\left(\frac{2.75 \times 1.96}{0.40}\right)^{2}=182
$$

This figure was increased, for the sake of safety, by about $20 \%$ and the final sample was thus determined to be 221 persons. This sample was selected for two main advantages: (1) it was small in relation to the size and therefore useful for carrying out further research; (2) it could assure the required reliability to draw some initial conclusions. The students' responses were not used in this final sample or in further analysis. The sample was distributed among the individual layers, based on the percentage of the population in each of the 12 layers in relation to the total population of the city. Following this, random sampling for the selection of the sample decided for each layer was done using the files of the National Statistical Service of Greece.

The questionnaire included 11 questions and was accompanied by the 32 coloured images. The questions had a multiple choice form and were selected in such a way as to describe the person questioned in relation to the environment he/she has lived in or is familiar with, during his/her contact with the countryside. Also basic demographic elements, such as sex, age, education, profession, income are included in the questionnaire. Each question corresponds to a single factor, generalised in a maximum of three categories. Besides, the relatively small size of the sample would not have benefited the larger in number categories. The questionnaire is presented in the Fig. 2. The eighth question was devoted to the scenic beauty of the various landforms presented in the coloured images. The responder was asked to rate the landforms on a 1-10 scale according to his/her preference. With regard to the ninth question about the profession, the third category entitled 'other' refers to unemployed, students, housewives and in general people who do not work.

The technique used for the questionnaire was interview-based. The main advantage of this technique is the direct contact with the person questioned and therefore presents the highest participation rate. The time available for the interview depends mainly on the way it is conducted. In this case, we decided to visit the persons questioned at home, to allow for a 10-15-min interview.

\subsection{Questionnaire results}

Table 1 shows the descriptive elements of the results' distribution, as derived for the scenic beauty of the various landforms. For the other questions the results are shown in Table 2. For the sake of brevity, all references hereinbelow will use the symbolism of factors shown in Table 2.

It is clear from the frequency values that there is sufficient number of observations in each category. The sample includes individuals for all the categories of age, education, income, profession and also individuals that come from different places of Greece or abroad.

As pertains to sex, women are the majority; however, the percentages are close to the respective percentages of the official census, in which men correspond to about $49 \%$ of the population and women to $51 \%$; therefore, these percentages are considered to be satisfactory for the balance between the two sexes.

As pertains to the scenic beauty, all images were rated with values from 1-10. The average responses for each image range within 5.02-7.72, with standard deviations from 1.91 to 2.62 . Therefore, the 


\section{Scenic Beauty of Various Landforms}

Please give a single answer to the following questions.

1. Sex:
1. [ ] Male
2. [ ] Female

2. Age:
$\begin{array}{ll}\text { 1. [ ] 18-35 years } & \text { 2. [ ] 35-50 years }\end{array}$
3. [ ] more than 50 years
3. You come from:
1. [ ] Athens
2. [ ] Outside Athens
3. [ ] Outside Greece

4. Where have you spent most part of your life?
1. [ ] Big city (
name of the city)
2. [ ] Small town (
name of the town)

5. How often do you visit the countryside?
1. [ ] Very often, almost every month
2. [ ] Not very often, two or three times every year

6. Usually for what purposes do you visit the countryside?
1. [ ] Leisure
2. [] Other

7. Using a scale of 1 (low preference) to 10 (high preference) how much would you evaluate the scenic beauty of the landform that is represented in each image?

Image 1:__ Image 2:__ Image 3:__ Image 4:__ Image 5:

Image 6:__ Image 7:__ Image 8: _ Image 9: _ _ Image 10:

Image 11: — Image 12: — Image 13: — Image 14: — Image 15:

Image 16: — Image 17: — Image 18: — Image 19:_— Image 20:

Image 21: — Image 22: — Image 23: — Image 24: — Image 25:

Image 26: — Image 27:_ Image 28: — Image 29:_ Image 30:

Image 31: — Image 32:

(n)

8. Education:
1. [ ] Primary
2. [ ] Secondary
3. [ ] Higher

9. Profession
1. [ ] Employees
2. [ ] Self-employed
3. [ ] Other

10. Income yearly
1. [ ] Less than 5 millions GRD
2. [ ] More than 5 millions GRD

11. Usually how long does your vacation last?
1. [ ] Less than 15 days
2. [ ] 15-30 days
3. [ ] More than 30 days

Figure 2: The questionnaire.

standard deviations are within the range of the values that had been observed from the experimental questionnaire to the students.

When observing the images in a descending order as to the mean scenic beauty, we can easily conclude that low values correspond to more plane forms, while high values to mountainous forms. As it has been already mentioned, this is one of the criteria used in landscape analysis and evaluation, 
Table 1: Descriptive elements of the distribution of results for the visual value of the landform of each image.

\begin{tabular}{rccc}
\hline Image & Mean $\bar{x}$ & Standard error $s(\bar{x})$ & Standard deviation $s$ \\
\hline 1 & 5.02 & 0.18 & 2.62 \\
2 & 6.81 & 0.16 & 2.42 \\
3 & 5.71 & 0.13 & 1.97 \\
4 & 5.49 & 0.14 & 2.06 \\
5 & 5.98 & 0.15 & 2.19 \\
6 & 6.28 & 0.13 & 1.95 \\
7 & 6.43 & 0.15 & 2.30 \\
8 & 5.67 & 0.17 & 2.48 \\
9 & 6.34 & 0.13 & 1.96 \\
10 & 7.05 & 0.15 & 2.28 \\
11 & 5.70 & 0.13 & 1.95 \\
12 & 6.02 & 0.15 & 2.21 \\
13 & 5.76 & 0.14 & 2.13 \\
14 & 5.56 & 0.16 & 2.38 \\
15 & 7.38 & 0.14 & 2.01 \\
16 & 6.51 & 0.14 & 2.09 \\
17 & 5.44 & 0.17 & 2.49 \\
18 & 7.41 & 0.14 & 2.14 \\
19 & 5.61 & 0.16 & 2.36 \\
20 & 7.49 & 0.14 & 2.06 \\
21 & 6.04 & 0.13 & 2.91 \\
22 & 6.59 & 0.13 & 2.00 \\
23 & 5.86 & 0.14 & 2.14 \\
24 & 6.10 & 0.14 & 1.98 \\
25 & 6.76 & 0.13 & 2.50 \\
26 & 5.86 & 0.17 & 2.00 \\
27 & 6.10 & 0.13 & 2.06 \\
28 & 5.86 & 0.14 & 2.95 \\
29 & 5.89 & 0.13 & 2.05 \\
30 & 5.68 & 0.15 & 2.01 \\
31 & 7.14 & 0.14 & \\
32 & 7.72 & 0.14 & \\
\hline
\end{tabular}

because mountainous forms present greater variety in the relief elements (slopes, curvatures, crest etc.) in relation to plane forms.

An interesting observation may also be derived, when we look at the mean values and the respective deviations of the answers. Let us consider that the standard deviation is the measurement of disagreement among the respondents, then we observe a greater disagreement of opinions in image 1 , and a greater agreement in image 21 . By observing the remaining images, it seems that disagreement tends to increase as the relief's visual value decreases. The respondents therefore seem to agree 
Table 2: Categories of factors.

\begin{tabular}{llc}
\hline Question/factor & General categories & Occurrence frequencies \\
\hline Sex (SEX) & Male & 93 \\
& Female & 128 \\
Age (AGE) & $18-35$ & 94 \\
& $35-50$ & 76 \\
Origin (FROM) & $>50$ & 51 \\
& Athens & 90 \\
Where have you spent & Outside Athens & 115 \\
most part of your life? (LP) & Outside Greece & 16 \\
How often do you visit the & Big city & 177 \\
countryside? (CVI) & Small town & 44 \\
For what purposes do you visit & $>1 /$ month & 65 \\
the countryside (RCVI) & $<2-3 /$ year & 156 \\
Education (EDU) & Leisure & 161 \\
& Other & 44 \\
Profession (PROF) & Primary & 61 \\
Time of vacation (HOL) & Secondary & 94 \\
Income (FIN) & Higher & 66 \\
& Employees & 85 \\
& Self-employed & 61 \\
& Other & 75 \\
& $<5$ mGRD & 151 \\
& $\geq 5$ mGRD & 70 \\
& $\leq 15$ days & 59 \\
& $15-30$ days & 122 \\
& $>30$ days & 40 \\
& &
\end{tabular}

more on what is good rather than bad. This observation is not an object of this study, but is presented as an interesting issue for further investigation.

\section{ANALYSIS OF VARIANCE - PRELIMINARY RESULTS}

The results were analysed with the analysis of variance for each individual factor [16]. The dependent variable was the scenic beauty of landforms. For any one interviewee there is only one set of factors which are repeated against all 32 images. Repetition of characteristics in multivariate analysis has unpredictable results. To avoid this problem and obtain some preliminary results, instead of using all the ratings given by each interviewee, the sum of ratings of all the images for each interviewee was used. In this manner the data matrix included 221 observations.

From the results gathered from this preliminary factor analysis of variance, we can draw some conclusions. In general, and in almost all the factors, the mean values of the individual categories differ from one another with a high degree of reliability. The $F$-ratio level of significance ranges from 0.00 to 0.0022 . With a probability of almost $100 \%$, this means that the average values of the individual categories are not equal. The higher the sum of the squares of errors between the groups, 
the better the factor classifies the dependent variable, and this sum can also become a benchmark for the factors. Factor analysis extracts factors which maximise the variance explained in order of the most important and so on. In descending order, the factors that were extracted are: LP, FROM, SEX, HOL, FIN, AGE, EDU, RCVI, PROF, CVI.

Considering the results of the analysis, the variation of the mean values in the categories of each factor leads to the following conclusions:

1. The persons who have spent most part of their lives in small towns or villages give higher ratings than those who have lived in cities or in city suburbs.

2. The persons who originate from areas outside Greece give ratings higher than those who come from areas outside Athens, and the latter give ratings higher than those who come from Athens.

3. Women give higher ratings than men.

4. The persons who spend more than 30 days annually for holidays give higher ratings compared to persons who spend 15-30 days, and the latter give higher ratings than persons who spend less than 15 days.

5. The persons with a family income below GRD 5,000,000 give higher ratings compared to those with a family income of more than GRD 5,000,000.

6. The persons aged 50 years or more give higher ratings compared to those in the $35-50$ age group and the latter give higher ratings compared to persons in the 18-35 age group.

7. The primary education graduates give higher ratings compared to the secondary education graduates, and the latter give higher ratings compared to university graduates.

8. Persons visiting the countryside for leisure purposes alone give higher ratings than persons visiting the countryside for other reasons.

9. Unemployed persons give higher ratings than self-employed and self-employed give higher ratings than employees.

10. The persons who visit the countryside more often than once a month give higher ratings compared to the persons who visit the countryside from time to time, 2-3 times a year or do not visit it at all.

The way the respondents used the same scale of values is different from person to person; however it seems feasible to group and generalise their behaviour. This is a first conclusion and perhaps one we would expect. What is that makes certain groups of people use higher values in relation to other groups? An important generalisation drawn from the observation is the following: the persons who have or had in the past more chances to come in contact with a physical environment use higher ratings. This probably explains why the persons who have lived in or come from the countryside, together with the persons who visit the countryside more often, the persons with more freedom in their work time, or the more aged persons who had more chances to visit the countryside, are the ones who know the physical environment better and thus give higher ratings. It is through the high degrees of freedom that they express their preference for nature.

The above observations are derived from the whole sample of the respondents. However, the fact that women gave higher ratings than men is a matter of concern and shows that we should perform a further investigation for both sexes separately. This will be the main concern of the following phase of this research, to find out whether the factors that affect each sex remain the same or not.

\section{DISCUSSION}

This study addresses the issue of the influence of the demographic data on the evaluation of the landforms' scenic beauty. It is clear from the results that the factors examined might influence to a certain extent the scenic beauty of the landforms. There could be many other factors that are even 
more important than the ones examined. No relative research had been conducted in the past so as to allow for a comparison. For example, perhaps the morphology of the place of origin of the respondents or the place where they have lived most of their life plays an important role in their preferences and evaluation, because each respondent is familiar, owing to their experiences, with certain relief forms. The investigation of such factors is not part of this study; however, it is a very interesting issue for further research, to better understand and interpret the public's preferences.

\section{REFERENCES}

[1] USDA Forest Service, National Forest Landscape Management, Ag. Handbook 434, US Government Printing Office: Washington, 1974.

[2] USDA Forest Service, Landscape Aesthetics, Ag. Handbook 701, US Government Printing Office: Washington, 1995.

[3] Kaplan, R., Kaplan, S. \& Wendt, J.S., Rated preference and complexity for natural and urban visual material. Perception and Psychophysics, 12(4), pp. 354-356, 1972.

[4] Kaplan, S., Some methods and strategies in the prediction of preference. Landscape Assessment - Values, Perceptions and Resources, eds E.H. Zube, R.O. Brush \& J.A. Fabos, Dowden, Hutchinson and Ross: Stroudsburg, PA, pp. 118-119, 1975.

[5] Daniel, C.T. \& Boster, S.R., Measuring landscape aesthetics: the scenic beauty estimation method, USDA Forest Service, Research Paper RM-167, 1976.

[6] US BLM, Visual Resource Management: Visual Resource Management Program, US Government Printing Office: Washington, 1980.

[7] Palmer, J., A visual character approach to the classification of backcountry trail environments. Landscape Journal, 2(1), pp. 2-12, 1983.

[8] Smardon, R., Palmer, J. \& Felleman, J., Foundations for Visual Project Analysis, John Wiley \& Sons: New York, 1986.

[9] Hunziker, M. \& Kienast, F., Potential impacts of changing agricultural activities on scenic beauty - a prototypical technique for automated rapid assessment. Landscape Ecology, 14(2), pp. 161-176, 1999.

[10] Tsouchlaraki, A., Digital relief visualisation in landscape analysis. Technika Chronika, Scientific edition of the Technical Chamber of Greece: I, 33, Athens, pp. 27-37, 1996 (in Greek with English extended summary).

[11] Cole, N., Ferraro, M., Mallary, R., Palmer, J. \& Zube, E., Visual Design Resources for Surface Mine Reclamation, Institute for Man and Environment/ARSTECHNICA, Center for Art and Technology, University of Massachusetts, Amherst, 1976.

[12] Tsouchlaraki, A., A Methodology for the Evaluation of the Visual Value of Natural Relief. PhD Thesis, Department of Rural and Surveying Engineering, NTUA, Athens, 1997.

[13] USDA Forest Service, The Visual Management System, Ag. Handbook 462, US Government Printing Office: Washington 1973.

[14] Damianou, Ch., Sampling Methodology - Techniques and Applications, Aithra: Athens, 1992 (in Greek).

[15] Koutsopoulos, K., Geography: Methodology and Spatial Analysis Methods, Symmetria Editions: Athens, pp. 367-422, 1990 (in Greek).

[16] Bora-Senta, E. \& Moysiadis, X., Applied Statistics, ZITI editions: Thessaloniki, 1992 (in Greek). 